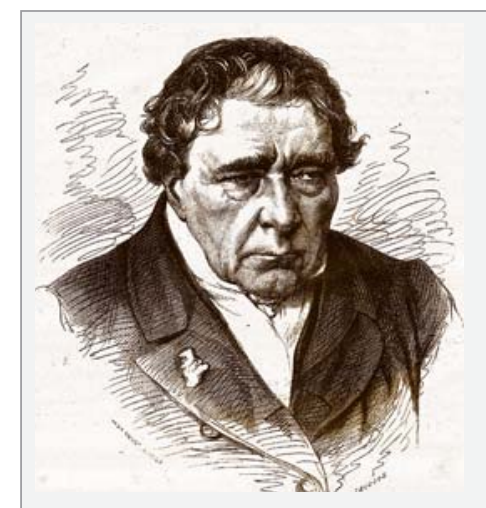

Principales dates

5 mars 1794 - Naissance à Lusignan, France

1820

1827

1831

1840

1854

Professeur de physique au collège Saint-Louis, Paris

Énonce le Principe de Babinet

Examinateur à l'École polytechnique

Membre de l'Académie des Sciences

21 octobre 1872 - Décès à Paris, France

\title{
Jacques Babinet
}

\section{Riad Haidar, haidar@onera.fr}

Mathématicien, physicien et astronome français, vulgarisateur talentueux, Jacques Babinet est surtout connu pour le principe d'optique qui porte son nom (selon lequel la forme d'une figure de diffraction est la même, en dehors de l'image géométrique, si elle est créée par un corps opaque ou par son « conjugué » obtenu en perçant une plaque aux emplacements où se situe ce corps). On lui doit plusieurs inventions ou perfectionnements $d^{\prime}$ instruments scientifiques, dont un polariscope, un goniomètre à collimateur pour la mesure des indices de réfraction, ainsi qu'un hygromètre... En cartographie, il a inventé le système de projection homalographique, qui conserve le rapport réel des surfaces.

Jisis acques Babinet naît à Lusignan, dans la Vienne, le 5 mars 1794. Sa mère, Marie, est la fille du lieutenant-général Bonneau du Chesne, maire perpétuel de Lusignan. Son père, Jean Babinet, est Conseiller au présidial de Poitiers, comme son propre père avant lui ; plus tard, il deviendra propriétaire du château du Murault, et occupera la charge de maire de Lusignan de 1812 à 1828.

Après de bonnes études littéraires au lycée de Poitiers, complétées par une année au lycée impérial Napoléon à Paris, Jacques entre en 1812 à l'École polytechnique. Onze mois après, il est envoyé à l'École d'application de Metz. II prend part en 1814, comme lieutenant au $5^{\mathrm{e}}$ régiment $\mathrm{d}^{\prime}$ artillerie à pied, à la défense de la ville face aux Alliés (troupes coalisées de la Russie, de la Prusse, du Royaume-Uni, de la Suède, de l'Autriche et de plusieurs États allemands). Rappelons que 1814 est l'année de la Campagne de France, qui s'achève sur l'abdication de Napoléon.

\section{Activités d'enseignement}

L'année suivante, le gouvernement de la Restauration décide de réduire l'effectif des armées ; Babinet abandonne alors la carrière militaire pour se vouer à l'enseignement. En 1816, il est régent en mathématiques au lycée de Fontenay-le-Comte en Vendée et publie, à tout juste 22 ans, son premier mémoire dans les Annales de Physique et de Chimie, intitulé Analyse mathématique sur les moyens de rendre sensible l'attraction solaire et lunaire par les variations du volume d'une masse de gaz soumise à une pression ne variant qu'avec la pesanteur. L'année suivante, après avoir obtenu le diplôme de bachelier es sciences sur son seul titre d'ancien élève de l'École polytechnique, il passe avec succès son examen de bachelier es lettres. Fort de ces grades académiques, il est nommé professeur de physique au Lycée de Poitiers.

En 1820, il devient professeur de physique au collège Saint-Lovis, qui vient d'être fondé à Paris, et épouse Adélaïde Laugier, la fille $d^{\prime}$ André Laugier, professeur et administrateur du Muséum d'histoire naturelle - union dont naîtront deux fils, Charles et Léon. II s'installe donc à Paris et côtoie les grands physiciens français de l'époque, parmi lesquels Fresnel, Arago ou Ampère. En 1822, il fait paraître avec Ampère un Exposé des nouvelles découvertes sur l'électricité et le magnétisme. En 1828, il succède à Fresnel au sein de la Société philomathique de Paris.

En 1831, Babinet entre dans le corps enseignant de l'École polytechnique comme examinateur, poste qu'il conservera plus de trente ans, successivement pour la géométrie descriptive, la physique et les sciences appliquées. À ce propos, l'un de ses élèves rapportera $\mathrm{qu}^{\prime}$ « il n'examinait pas comme tout le monde et s'attachait moins à constater si l'élève avait retenu plus ou moins fidèlement ses cours qu'à reconnaître si cette jeune intelligence saurait un jour tirer parti de l'instrument qu'on lui avait mis entre les mains, quand l'usage lui en aurait facilité le maniement » [1]. Fort de son expérience d'enseignant, Babinet publie en 1851, sur la demande de l'éditeur Hachette, un Traité élémentaire de géométrie descriptive qui fera longtemps partie des livres de préparation au concours d'entrée à l'École polytechnique. 


\section{Travaux scientifiques}

On doit à Babinet de nombreux travaux originaux sur l'optique et sur les phénomènes météorologiques. Sa carrière se distingue particulièrement par les nouveautés qu'il introduit dans le domaine de l'instrumentation scientifique. En 1824, il équipe l'hygromètre de Horace de Saussure, destiné à mesurer l'humidité de l'air, $d^{\prime}$ 'un système de lecture par microscope et vis micrométrique qui en améliore sensiblement les performances. En 1827, s'inspirant des travaux de Fraunhofer sur la diffraction de la lumière par les réseaux, il montre que l'observation des franges d'interférence permet d'étalonner les longueurs de façon très précise, suggestion qui mènera plus tard à la définition du mètre à partir de la longueur d'onde d'un rayonnement des atomes de krypton. Il énonce également le principe d'optique qui porte son nom, selon lequel deux écrans d'ouvertures complémentaires produisent la même figure de diffraction.

En 1830, l'Académie royale des sciences le récompense pour un perfectionnement important de la machine pneumatique de Robert Boyle qui permet d'obtenir un vide plus poussé sous une cloche de verre. En 1839, il réalise un nouveau type de goniomètre destiné à mesurer les angles dièdres des cristaux; portable et maniable, il permet aussi de mesurer la déviation de la lumière par un prisme et sera utilisé vers 1860 par Kirchhoff et Bunsen pour une analyse spectroscopique des corps introduits dans une flamme.

Babinet publie de nombreux mémoires dans les Annales de physique et de chimie, de 1824 à 1841, et dans les Comptes rendus de l'Académie des sciences, de 1837 à 1865. On lui doit également un nouveau système de projection, dit homalographique ou à parallèles rectilignes, dans lequel les cercles parallèles sont représentés par des droites et les méridiens par des ellipses : pour dresser les cartes, ce système permet d'établir une proportionnalité exacte entre des surfaces quelconques de la Terre et les parties correspondantes de sa représentation.
Excellent pédagogue, Babinet supplée Ampère au Collège de France en 1832, et y enseigne l'optique. En 1839 et 1840 il supplée Savart, toujours au Collège de France, puis Pouillet en 1846, cette fois à la Sorbonne, et y dispense les leçons d'acoustique et d'optique. Babinet est élu à l'Académie des Sciences en 1840, où il succède à Dulong, chimiste et physicien français, auteur de travaux sur la dilatation et l'indice de réfraction des gaz. II en devient I'un des membres les plus assidus, jusqu'à sa mort. En 1854, il est nommé astronome-adjoint au Bureau des Longitudes. Par ailleurs, il entretient pendant 40 ans (de 1829 à 1869), une correspondance suivie avec l'illustre astronome anglais John Herschel.

\section{Ultima verba}

Plus encore que par ses qualités d'enseignant, Babinet acquiert une formidable popularité par le talent avec lequel il sait vulgariser la Science. Ses lectures aux solennités annuelles de l'Institut, ses articles insérés dans la Revue des Deux Mondes ou le Journal des Débats, ses cours à l'Athénée et ses conférences publiques à l'École de Médecine, sous les auspices de l'Association polytechnique, sont tous très appréciés du grand public. Possédant une culture littéraire exceptionnelle, doué d'une mémoire prodigieuse, il sait captiver l'attention et rendre agréables les sujets les plus arides par des rapprochements inattendus et de piquantes anecdotes.

Il est à Paris en 1870, et subit les privations du siège de la ville, ainsi que les tristes scènes de la Commune. Ces événements lui portent un coup terrible dont il ne parvient pas à se remettre : il rend l'âme le 21 octobre 1872, à Paris.

\section{Pour en savoir plus}

[1] Albert de Rochas, Jacques Babinet, in Le Livre du Centenaire de l'École polytechnique (1897).

[2] Bernard Pire, Jacques Babinet (1794-1872), Encyclopædia Universalis.

\section{SPECTROGON}

\section{Filtres Interférentiels}

De 200 á $15000 \mathrm{~nm}$

- Passe-bande

- Passe-haut

- Passe-bas

- Large bande

- Densité neutre

- Disponible en stock

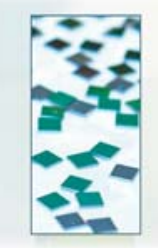

\section{Réseaux Holographiques}

De 150 á $2000 \mathrm{~nm}$

- Compression d'impulsion

- Télècom

- Accordabilité spectrale

- Monochromateurs

- Spectroscopie

- Disponible en stock 